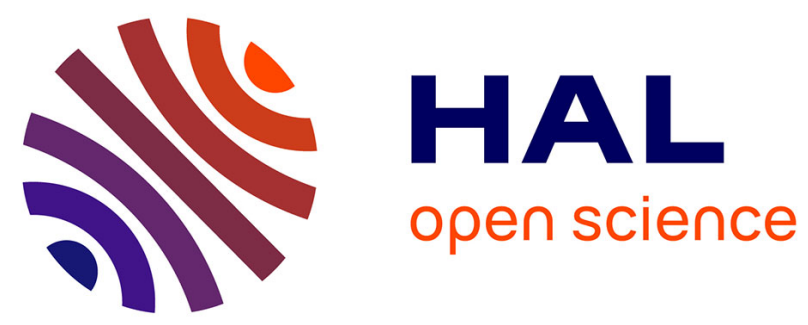

\title{
Les arts-de-faire du terrain
}

Chloé Buire

\section{To cite this version:}

Chloé Buire. Les arts-de-faire du terrain. "À travers l'espace de la méthode: les dimensions du terrain en géographie", Arras, 18-20 juin 2008, Jun 2008, Arras, France. halshs-00421957v2

\section{HAL Id: halshs-00421957 https://shs.hal.science/halshs-00421957v2}

Submitted on 12 Oct 2009

HAL is a multi-disciplinary open access archive for the deposit and dissemination of scientific research documents, whether they are published or not. The documents may come from teaching and research institutions in France or abroad, or from public or private research centers.
L'archive ouverte pluridisciplinaire HAL, est destinée au dépôt et à la diffusion de documents scientifiques de niveau recherche, publiés ou non, émanant des établissements d'enseignement et de recherche français ou étrangers, des laboratoires publics ou privés. 


\title{
Les arts-de-faire du terrain
}

\author{
Chloé Buire \\ Doctorante en géographie \\ Laboratoire Géographies Comparées des Nords et des Suds (GECKO) \\ Université Paris Ouest - Nanterre
}

Communication au colloque "À travers l'espace de la méthode : les dimensions du terrain en géographie", Arras, 18-20 juin 2008

Lorsque l'appel pour le colloque de 2008 a été publié, j'étais en plein 'terrain', à Cape Town en Afrique du Sud ${ }^{1}$. Mes progrès en anglais et l'habitude de conduire à gauche commençaient à rendre mes journées plus 'professionnelles' : immersion auprès de différentes familles, participation à des réunions municipales, entretiens ciblés avec certains responsables. Comment s'articulent les institutions post-apartheid avec l'héritage politique de cinquante années d'oppression ? Depuis la nomination de Nelson Mandela à la tête du pays en 1994, l'Afrique du Sud se taille un nouveau costume démocratique à grand renfort de réformes institutionnelles. L'ouverture au capitalisme mondialisé rend l'opération délicate : comment prendre les mesures d'un corps social marqué par des transformations accélérées (croissance urbaine, chômage, criminalité...) et des inerties préoccupantes (niveau de l'éducation publique, organisation raciale des villes, modèle agricole latifundiaire...)?

Invitée à interroger les 'dimensions cachées' du travail de terrain, je me suis rendue compte que le débat sur les valeurs du 'terrain' était au coeur de ce qui se jouait dans les arènes politiques que j'explorais jour après jour. La rhétorique politique, administrative et scientifique concernant la démocratisation sud-africaine fait sans cesse appel à la notion de ground: les people on the ground sont la cible de l'action publique et leur soutien est une condition sine qua non du 'développement' et de la 'bonne gouvernance' urbaine. De la même manière, ma posture de recherche privilégiant l'entrée par le micro-local et donc la parole des people on the ground repose sur la thèse qu'une analyse ethnologique 'par le bas' permet une lecture scientifique des dynamiques sociales à la portée plus générale.

Mais alors que la littérature dénonce les dérives du 'mythe du ground', décriant le populisme et le clientélisme dans la prise de décision et la distribution des ressources, le chercheur semble généralement peu inquiet des glissements possibles de son propre positionnement. Sa place particulière d'enquêteur extérieur au corps social qu'il analyse est pourtant elle aussi ambiguë : quelles sont les garanties de sa propre responsabilité vis à vis de ses 'objets' ? Comment négocie-t-il sa légitimité sur le terrain et dans le champ scientifique à la fois?

Cette étude de cas est une métaphore de la nature irrémédiablement politique des relations 'de terrain' qu'il serait vain de prétendre dépasser au nom de l'objectivité scientifique. Qu'il le veuille ou non, le chercheur est pris à parti dans ses relations quotidiennes et il lui revient d'intégrer cette dimension, non seulement dans ses tactiques méthodologiques mais également dans le savoir qu'il a vocation à produire in fine.

\footnotetext{
Cet article repose sur un travail de thèse en cours dirigé par Philippe Gervais-Lambony et financé par une allocation ministérielle. Un immense merci à tous ceux qui le rendent possible au quotidien. Un merci plus grand encore à mon directeur pour sa patience et ses encouragements.
} 
En fin de compte, j'ai fait de l'invitation de l'équipe du colloque 'Terrain' l'opportunité d'imaginer le rôle social du chercheur comme un exemple d'humanisme sensible où l'improvisation pragmatique l'emporte sur le calcul stratégique, à l'image des savoirs-faires déployés au quotidien par les citadins pauvres de Cape Town.

\section{Visions sud-africaines : Le mythe du 'ground' au coeur du débat sur la citoyenneté post-apartheid}

À travers les débats irrésolus qui traversent ma thèse, je souhaite d'abord rappeler certaines ambiguïtés liées à la question du 'terrain' comme matrice de légitimité du discours. En Afrique du Sud et en particulier dans les métropoles de ce pays émergent, la construction de la démocratie après cinquante ans d'apartheid est profondément ancrée dans l'idée du 'ground'. D'une part, la lutte contre le gouvernement raciste a nourri une culture politique fondée sur les liens directs au sein des communautés locales. D'autre part, les impératifs de la 'bonne gouvernance' imposent une prise en compte de plus en plus formelle des voix citadines. Mais aujourd'hui, ces relations individualisées sont vivement dénoncées en ce qu'elles sont le ferment d'une gestion politique clientéliste qui handicape l'émergence tant espérée d'une société multi-raciale égalitaire.

À travers l'immersion dans les communautés de Gugulethu et d'Heideveld, à travers les entretiens approfondis avec les familles précaires de ces deux townships de Cape Town, le travail de terrain met en évidence la complexité des débats sur la citoyenneté post-apartheid. autogérées

Le double héritage de l'apartheid : un corps social fragmenté, des communautés locales

Parmi ses nombreuses obsessions totalitaires, le régime d'apartheid est célèbre pour ses lois racistes encadrant la croissance urbaine. Le principe de la ségrégation urbaine repose sur le double jeu entre la manipulation de l'espace et la manipulation des identités. D'une façon générale, les individus étaient niés dans leur libre-arbitre pour être réduits à des statistiques raciales. Les nonBlancs en particulier ont été exclus des droits civiques, expulsés et regroupés dans des quartiers périphériques marqués par un urbanisme de contrôle : les townships.

\section{Loin du centre, loin de la montagne, loin des plages}

Les townships constituent un terrain de recherche 'hors la ville' par rapport aux clichés de la Ville Mère du tourisme sud-africain. Le ward 44 est la circonscription électorale qui regroupe les quartiers étudiés de Heideveld et Gugulethu.

Rue principale de Gugulehtu. Son nom 'NY 1' cache l'appellation d'origine 'Native Yard 1' (cliché C. Buire, février 2007)

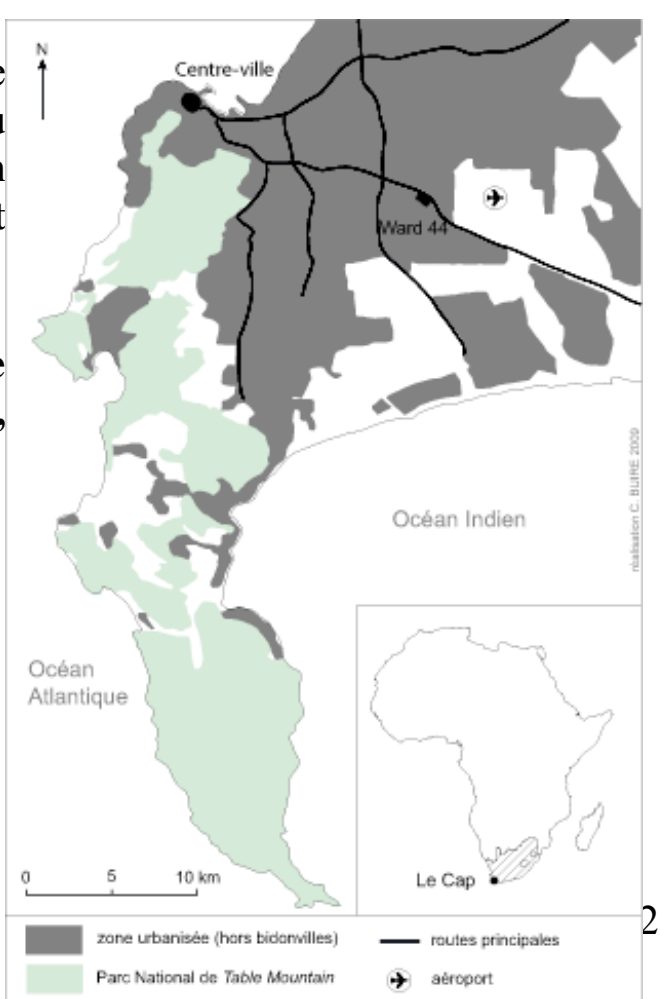




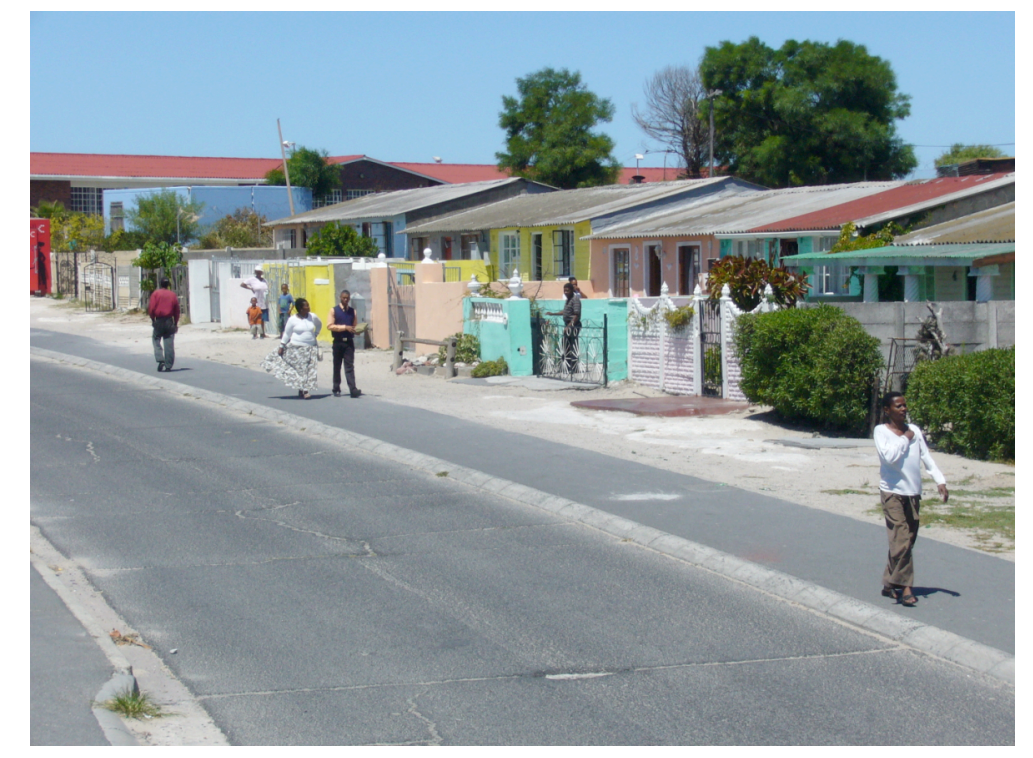

Depuis le Native Urban Areas Act de 1923 qui a institué le principe du passeport urbain pour les hommes africains considérés comme étrangers à la ville jusqu'à la loi sur les 'Autorités Locales Noires' en 1983, le gouvernement s'est efforcé de rejeter les non-Blancs en dehors de la ville. Le besoin de main d'oeuvre a pourtant conduit à la construction de townships, quartiers résidentiels aux infrastructures minimales, où les seuls lieux publics sont des lieux contrôlés : police, écoles, stades. Aujourd'hui encore, le paysage de Gugulethu est marqué par l'uniformité des 'maisons-boîtes d'allumettes', révélatrices du minimalisme dans lequel étaient tenues les populations non-blanches.

Une série de lois a donc tenté pendant un demi-siècle d'inventer une sous-citoyenneté pour les habitants des townships. Le bilan de ces efforts est à l'opposé du résultat escompté : les communautés 'non-blanches' ont inventé des vies citadines 'hors-la-ville' et ont développé des compétences politiques autonomes. Cette solidarité de survie a progressivement été relayée et prise en charge par les institutions clandestines des mouvements de résistance au régime d'apartheid. Sans même évoquer les grandes actions de la Lutte (révolte des étudiants de Soweto en 1976, boycotts généraux des années 1980 etc.), l'auto-organisation s'est concentrée sur les problèmes quotidiens (logement, santé, éducation), et s'est ancrée dans des cellules communautaires regroupant quelques centaines de voisins : Civics, branches locales de l'ANC, 'area committees' ${ }^{2}$.

La vie dans les townships est ainsi associée à une culture de la participation populaire. La familiarité est un trait majeur des relations politiques, familiarité au sens propre, étendue à l'ensemble de la communauté. Le principe élémentaire de la démocratie comme 'pouvoir du peuple' fait donc écho à un sens du politique spécifique au cœur des débats sur la démocratisation sudafricaine. Décrivant l'émergence comparable d'une sphère alternative au sein de la société civile brésilienne, J. Holston (2008 : 248) propose la notion de 'citoyenneté insurgée' ('insurgent citizenship') pour décrire ces processus de démocratisation par le bas :

"In the insurgent formulation, the residents of the periphery imagine that their interests derive from their own experience, not from state plans, that they are informed and competent to make decisions about them, and that their own organizations articulate them. They consider this organized experience the basis for an exercise of citizenship through which they participate in and hold accountable the institutions of society, government, and law that produce the conditions of urban life.»"

\section{« EVERYTHING COMES FROM THE GRASSROOT LEVEL »}

Dans cet extrait d'interview (19 décembre 2008), Rose exprime son attachement fondamental au principe d'une démocratie venue du 'ground' et la frustration profonde face au

L'ANC, African National Congress, est le parti de Mandela, banni sous l'apartheid et aujourd'hui majoritaire au gouvernement. Les Civics, et les 'area committees' sont tous d'une façon ou d'une autre reliés à l'ANC. Transformer les réseaux informels de la résistance en fonctionnement démocratique constitue la toile de fond des relations politiques sud-africaines. 
fonctionnement de la démocratie représentative qui repose, de fait, sur la distinction entre l'élite dirigeante et la base populaire.

"... they are top guys okay and you can never be a top guy and never be elected by the grassroot level. Everything comes from the grassroot level. So that what we are complaining of. Because they take the decisions, they finalize everything. They don't come down to, you know, to people like locally

(...)

And they, by their say 'people shall govern' (...). But you know, it's not 'people-who-shallgoverning' now. ANC is governing, you know. And it's not all the ANC. SOME of the ANC: LEADERS, not people, LEADERS are governing. Which is very unfair. "

Plus tard, Rose souligne sans le vouloir le jeu ambigu des relations politiques individualisées. L'ampleur du remaniement des élites politiques après l'apartheid a conduit au pouvoir des individus qui vivaient jusqu'alors dans les townships et dont l'éducation politique reposait sur le quotidien de la Lutte, expérience largement partagée parmi la majorité non-blanche.

"Chloe you won't believe it, fortunately people who are in the ANC, for instance the general secretary we grew up together. We were staying together, we were seeing each another, but he will never ever... Even with the previous Mayor of Cape Town, we were together at school, we were even doing catering for the Gugulethu library. We did pass our business card. What do they do? Nothing! »

Rose espérait donc plus de soutien de la part de ses anciens camarades, mais au lieu de cela, 'ils ne font qu'aider leur famille'. Lucas, son mari, ne peut se retenir de se joindre à la conversation :

«Lucas : Sorry lady, what they do...

Rose, le coupant : they keep their families....

Lucas : ... what they do, with those tenders, even they see she [Rose] is doing something, catering. She [former Mayor] will teach HER family to do catering. And give those...

Rose, le coupant : everything is corrupt...

Lucas : ... give those to their family, not to the people.

Rose : everything is corrupt.

Lucas : ... only their families! And you see their family drives in Porsche cars and those things, listen, beautiful houses, but we can't do nothing! Why do they get the tenders and yet they are working? Why not give the people who are not working the tenders? "

Si l'on raisonne selon des principes éthiques, il ne semble pas plus juste qu'un ministre privilégie ses anciens camarades ou sa famille. Il est de toute façon sensé servir tous les citoyens sans distinction. Un politicien aguerri ne commettrait pas cette maladresse, mais ici le ton est à la conversation familière. Le politiquement correct n'a pas lieu d'être. Rose aurait bien aimé profiter de sa familiarité avec le Maire, même si cela entre en contradiction avec son idéal théorique de justice et de démocratie. Ce décalage souligne l'importance idéologique du mythe du 'ground' : la démocratie est synonyme de prise en compte des besoins de chaque individu. Pour Rose, cela signifie réaliser quelques bénéfices grâce à sa compagnie de traiteur, afin de payer le lycée de sa dernière fille. Mais comment délimiter la légitimité des besoins de chacun ? Pour certains, conduire une Porsche fait partie du minimum qu'ils peuvent offrir à leurs enfants... À un moment ou à un autre, il faut bien que le chercheur pose des jalons idéologiques à ses propres interprétations. 
Alors que le marketing urbain déploie des slogans un brin surannés aux yeux du chercheur ('This City Works For You' pour la métropole capetonienne, 'We Are The Same' à Pretoria), les discours des citadins rappellent que la vitalité démocratique sud-africaine prend bel et bien ses racines dans une histoire et une culture politique où l'existence de la communauté et la volonté individuelle sont les deux faces d'un même système de valeur. Ubuntu, 'je suis parce que nous sommes'. Il y a donc un malaise latent dans l'interprétation scientifique. Au nom de quoi peut-on décider de ce qui est 'naïf' ou au contraire 'important' ?

\section{Clientélisme et populisme, les ambiguïtés irrésolues de la démocratie 'par le bas'}

La définition de la Démocratie comme expression directe de la volonté de chacun est un mythe fondateur pour la Nation Arc-en-Ciel. Un mythe qui a des vertus thérapeutiques pour un corps social déchiré par le racisme et la violence de l'apartheid mais qui risque d'aboutir à un populisme cynique dans la mesure où c'est désormais le gouvernement légitime qui utilise la rhétorique du 'mouvement populaire de masse'. De nombreux analystes radicaux dénoncent cette mise sous tutelle de la société civile. L'enthousiasme militant de 'l'ancien temps' est instrumentalisé. Les réunions clandestines où chacun venait contribuer à la survie collective quotidienne, les grandes manifestations où seul l'effet de masse promettait un peu de protection contre la répression armée deviennent des farces où le peuple se doit d'applaudir ses dirigeants. Depuis la libération de Nelson Mandela, le pays a gagné une place de choix dans le concert des Nations. L'ère de l'opprobre internationale a fait place à l'ouverture au capitalisme mondial. Comme dans tous les pays émergents, l'afflux de capital et la compétition génèrent des écarts socio-économiques croissants. C'est la juste distribution des ressources nationales qui anime les revendications fondamentales et non plus l'accès aux droits civiques. L'utopie sociale devient sonnante et trébuchante. La familiarité des camarades est alors de plus en plus lue comme preuve de clientélisme. Les scandales éclatent et s'étouffent à toutes les échelles.

\section{CAPE TOWN COMMUNITY HOUSING COMPAGNY, ODYSSEE D'UNE TRIVIALE AFFAIRE DE CORRUPTION}

L'exemple de la CTCHC (Cape Town Community Housing Compagny) illustre l'écheveau des lobbys dans le secteur de la construction. La compagnie a pour vocation la construction de logements subventionnés par l'Etat. Dans le principe même finances publiques et bénéfices du privé sont associés. Depuis sept ans maintenant, la compagnie est poursuivie en justice par un collectif d'habitants qui dénonce le projet de neuf lotissements réalisés dans divers townships de Cape Town à la fin des années 1990. Leurs plaintes concernent à la fois la qualité matérielle des maisons et les zones d'ombre de leur financement. Les murs sont faits de plus de sable que de ciment. Le soustraitant a expliqué que pour contrebalancer les versements indigents de la Compagnie, il a revendu une partie du ciment. La Compagnie avance que les pressions politiques l'ont forcée à privilégier le calendrier à la qualité. De plus, les avocats ont prévenu les habitants : ils ne sont pas prêts d'obtenir leur titre de propriété, la compagnie n'est toujours pas officiellement propriétaire de ses terrains. Les maisons n'apparaissent pas sur le cadastre. Les traites mensuelles établies à 350 rands dans les plans d'épargne initiaux ont grimpé à 800 rands après la remise des clés. Les explications à ce sujet sont encore plus floues mais les habitants ont eu recours à leur seule arme, aiguisée dans les années d'apartheid : le boycott des loyers. Après plus de huit ans d'un conflit quasi-quotidien, les ordres d'expulsion arrivent dans les boîtes aux lettres et ravivent le scandale. En mai 2009, le nouveau maire hérite de ce dossier parmi des centaines d'autres. Les habitants des lotissements de la CTCHC ne se font aucune illusion sur un dénouement possible. Le maire fait partie du board of directors de la compagnie... 
Le scandale de la $\mathrm{CTCH}$ est un autre exemple d'objet délicat à manier pour le chercheur. Il y a d'abord de nombreux obstacles pour démêler le conflit : qui écouter ? qui croire ? Les membres du comité des habitants me font régulièrement lire les lettres de la Compagnie, les échanges de mails photocopiés entre leur avocat et les autorités provinciales, les coupures de presse mais que faire de ce matériau assurément partial ? J'ai l'impression de ne jamais en savoir suffisamment mais en même temps, en apprendre plus, c'est aussi changer de rôle et devenir partie prenante des échanges.

J'ai choisi de trancher la question en laissant le conflit au rang de simple objet de recherche, un objet parmi d'autres pour explorer un sujet qui n'est pas celui de la corruption mais celui de la citoyenneté telle qu'elle est mise en discours et en pratique au quotidien. C'est finalement renoncer à saisir la totalité de 'l'affaire'. En acceptant de suivre les discours fragmentaires plutôt que 'le réel', j'ai pris parti pour les tactiques potentiellement irrationnelles de ceux qui fabriquent la ville au jour le jour, ce que Béatrice Collignon (1996) a choisi d'appeler les 'savoirs vernaculaires' :

«Le savoir géographique apparaît sous la forme d'une construction éphémère sans cesse recomposée, d'un moment dans la pensée plus que d'une structure permanente. (...) ainsi ce savoir n'est qu'une matrice, un mode d'articulation des connaissances qui n'existe en permanence qu'à l'état latent et ne s'actualise que dans la situation qui implique sa mobilisation. »

\section{Résoudre les paradoxes de la recherche de 'terrain' en adoptant les arts-de-faire 'on the ground'}

La définition de savoirs 'vernaculaires' donnée par Béatrice Collignon permet de dépasser les apparentes contradictions de bien des situations. Ainsi à Station, un quartier dégradé de Heideveld où la CTCH a bâti un lotissement en 2000, le pasteur de l'Eglise évangéliste fabrique son leadership communautaire en multipliant les registres de légitimité. Combat social, exégèse protestante, prise de position politique apparaissent comme un ensemble de connaissances éparses que l'on peut toutefois comprendre comme l'expression d'un savoir politique vernaculaire si l'on s'en tient à l'idée qu'un tel savoir " ne s'actualise que dans la situation qui implique sa mobilisation ».

La problématique est ainsi légèrement déplacée : il ne s'agit pas de trancher le débat sur le clientélisme et la corruption mais d'observer comment s'élabore le leadership local. Bien que le contexte brésilien ne puisse être plaqué sans précaution sur le cas sud-africain, le texte de J. Holston (2008 : 248) aide à dessiner les contours d'une analyse politique ancrée dans les expériences communautaires quotidiennes.

" The key is not whether people accept a load of bricks from an aspiring candidate (who would not?) or vote for those candidates who deliver them (a rational choice of interest). Nor does it seem useful to multiply analytic categories of clientelism as a means to gauge whether interests are more or less 'emptied of their political content', as if interests (not to mention government provision of resources) could ever be apolitical.

Rather, the key is whether alternatives exist to direct and coerced exchange of resources for political support. The sphere of civic participation that the residential organizations created established this alternative. »"

\section{LEADERSHIP COMMUNAUTAIRE, LA CONFUSION DES REGISTRES POLITIQUES}

Le pasteur est engagé dans le conflit avec la CTHC depuis le début. En l'espace de huit ans, il a expérimenté une multitude d'attitudes possibles avec la compagnie allant du conflit ouvert à travers des actions illégales (occupations des logements avant la remise des clés, boycotts des loyers) jusqu'à la collaboration officielle sous la forme de contrats de 'CLO' (community liaison 
officer). Le pasteur s'est progressivement imposé comme leader de sa communauté, reconnu comme personne ressource à la fois par les voisins et par les décideurs locaux. Cette double légitimité est un critère essentiel pour identifier un 'leader'. Mais comment mesurer cette reconnaissance?

Le temps passé à Heideveld permet de saisir certaines de ces occasions où l'autorité du leader est mise en pratique : célébrer les funérailles d'une voisine assassinée, organiser une collecte d'argent pour les personnes âgées, participer au réseau des parents d'élèves de l'école du quartier sont autant de moments a priori triviaux et pourtant déjà performatifs du lien politique. L'église, le centre communautaire, l'école sont des lieux quotidiens qui ont leur fonction propre mais ils peuvent être analysés dans leur dimension politique au gré des évènements qui s'y célèbrent. Un office d'enterrement sort de la banalité lorsqu'il soulève le débat sur la criminalité et la sécurité du quartier, la kermesse caritative implique une coordination avec les autres organisations susceptibles de lever des fonds, le conseil des parents d'élèves a une indéniable responsabilité sociale (prévention contre le SIDA ou l'usage de drogues, intégration raciale des élèves venus d'autres quartiers etc.). C'est ainsi que les registres de légitimité se superposent pour tisser l'épaisseur politique du pasteur.

Lorsqu'approchent les élections, le pasteur fait campagne pour le Parti Chrétien Démocrate Africain $(A C D P)$ avec autant de passion que pour ses sermons dominicaux. L'autorité morale de l'Eglise devient partisane. Tous les deux ou trois mois, le pasteur est officiellement invité à commenter l'agenda politique de sa circonscription en tant que membre du comité consultatif des électeurs du ward 44. Les savoir-faire acquis dans le conflit avec la Compagnie sont utilisés pour faire des propositions aux conseillers municipaux...

Les décrets qui encadrent la participation locale plaident pour la dépolitisation des habitants. Aux yeux de l'administration, utiliser les institutions municipales pour défendre des intérêts personnels est une grave atteinte à la qualité du service public. Mais comme le montre le travail de terrain, cette dépolitisation est un vœu pieux bien éloigné des réalités quotidiennes. Choisir au contraire une lecture ancrée dans les pratiques permet de dépasser ces impasses normatives.

Les séjours répétés au Cap, les heures passées avec le pasteur de Station nourrissent une familiarité caractéristique des 'terrains ethnologiques'. Il s'agit maintenant de souligner comment cette posture méthodologique influence le savoir scientifique lui-même. Il est probable que Rose n'aurait pu exprimer ses interrogations politiques autrement que dans le cadre d'un entretien très libre avec une personne familière. Les situations d'enquête informelles produisent un matériau hétéroclite plein de contradictions. Or ce sont bel et bien ces contradictions qui permettent de nourrir le débat sur le sens du politique au niveau micro-local. La valeur de la participation politique, si profondément intériorisée se heurte régulièrement à un désarroi sincère face au fonctionnement d'une démocratie institutionnalisée. C'est ce que montrent aussi les ambiguïtés du leadership du pasteur. S'engager pendant des années dans une bataille judiciaire contre une compagnie dûment protégée politiquement n'empêche pas d'accepter des contrats de cette même compagnie. Le conflit est mené au nom de la protection des intérêts communautaires, mais en même temps le poste de CLO participe à construire l'image du pasteur comme représentant de cette communauté.

La valeur heuristique du terrain est partagée par J.Holston, sa formulation des ambiguités de la citoyenneté insurgée 'montre les limites d'une théorie de la démocratie fondée sur des conceptions du politique à la foi étroites et totalisantes et suggère les avantages d'une vision anthropologique qui considère les conditions de la citoyenneté, dans leur vécu et leur contingence - et malgré leur dimension désordonnée, désobéissante - comme un meilleur moyen pour comprendre les formes 
contemporaines du développement de la démocratie. ${ }^{3}$

\section{Théorie des opportunités politiques : Les arts de faire populaires ne relèvent pas de stratégies}

Réfléchissant à la nature du savoir produit par les sciences sociales, Michel de Certeau a proposé une distinction entre les stratégies et les tactiques. L'ouvrage qu'il a co-écrit avec Luce Giard et Pierre Mayol propose un cadre théorique selon lequel le défi élémentaire du chercheur repose sur la différence essentielle entre deux types de savoir : la rationalité scientifique qui relèverait d'un 'modèle stratégique' d'une part et les 'arts de faire' du quotidien, qualifiés de 'tactiques' d'autre part. Pour reprendre les termes de M. de Certeau (1990 : xlvi-xlvii), la tactique est de l'ordre du 'calcul' :

«... il lui faut constamment jouer avec les évènements pour en faire des "occasions" (...) des moments opportuns où [elle] combine des éléments hétérogènes [dont la] synthèse intellectuelle a pour forme non un discours, mais la décision même, acte et manière de "saisir" l'occasion. »

Cette définition de la tactique fait écho à la description des savoirs vernaculaires proposée par Béatrice Collignon et à l'opportunisme pragmatique décrit à propos du pasteur. La confrontation aux paradoxes qui caractérisent les péripéties des défis quotidiens, les 'bread and butter issues', permet donc une alternative au débat insoluble qui oppose la rhétorique utopiste 'du ground' au pragmatisme désenchanté d'une théorie du clientélisme. L'insaisissable caractère des relations sociales oblige à réviser la vision stratégique du discours scientifique pour adopter l'image du rhizome utilisée par G. Deleuze et F. Guattari : les registres identifiés en théorie se confondent dans le discours d'un même individu, les acteurs sont tous liés dans une même matrice sociale sans qu'il soit possible d'établir un point de départ et un point d'arrivée. Et le chercheur lui-même ne fait pas exception, il fait partie de cette 'rhizomatique'...

Comment le chercheur se maintient-il en équilibre dans cet ensemble ? Dans quelle mesure construit-il son propre discours, recevable à la fois scientifiquement auprès de ses pairs ET socialement dans le groupe auquel il s'intéresse?

\section{"Peut-être qu’à recourir aux procédures mêmes de cet art... " (M. de Certeau)}

« D'une part : cet « art », au nom de quoi le disons-nous différent D'autre part : d'où (de quelle place distincte) procédons-nous à son analyse ? Peut-être qu'à recourir aux procédures mêmes de cet art, nous pouvons réviser et sa définition comme « populaire » et notre position d'observateur. » (de Certeau, 1990 : xlvii).

En interrogeant le modèle de la rationalité scientifique, Michel de Certeau invite à abolir la distinction entre la voix du chercheur et celle de ceux qu'il interroge. La proposition est séduisante sur le plan théorique, comment lui donner corps à travers l'exemple d'un terrain de thèse?

Ma thèse prétend décrire les arts de faire du politique dans les townships. Mais tout ce que je sais de ces arts de faire, je l'ai appris en les mettant moi-même en pratique. Ce que j'ai à dire sur la citoyenneté, je le dois à mes propres tactiques, à mon apprentissage au jour le jour de ce qu'il faut dire, à qui, à quel moment, à quel endroit etc. Pour comprendre les relations politiques, il a fallu

'It shows, moreover, the limitations of democratic theory based on narrow yet totalizing conceptions of the political an suggests the advantages of the anthropological view that considers the lived and contingent conditions of citizenship - however messy and disjunctive - as a better means of understanding contemporary form of democratic development.' (Holston, 2008 : 275) 
être capable de participer à leur cercle. De devenir un acteur dans ce système. Et il ne s'agit pas de renoncer à la position de chercheur en montant un projet communautaire ou en commençant une carrière politique mais d'accepter que 'être chercheur' est un rôle social en soi. "La jeune fille française que l'on voit régulièrement aux réunions municipales, celle qui conduit cette voiture bleue souvent garée devant la maison du pasteur, cette fille-là s'intéresse à ce qui se passe chez nous. » Certains sont méfiants, d'autres espèrent y trouver quelque chose à gagner. Dans tous les cas, la présence d'un chercheur doit être considérée comme une perturbation à travers laquelle le corps social se révèle, mais une perturbation parmi tant d'autres, avec pour particularité de proposer un échange gratuit, fondé sur la curiosité mutuelle et la communication avant tout.

Le travail de terrain prend alors une dimension esthétique. Il s'agit d'un apprentissage humain fondé sur des qualités d'improvisation. Certains essais sur la musique, et sur le free jazz en particulier permettent de pousser un peu la métaphore. Improviser, ce n'est pas seulement laisser libre cours à son imagination dans une spontanéité 'naturelle'. En jazz, improviser requiert une grande maîtrise des codes musicaux. Le musicien doit connaître la structure harmonique, la suite d'accords sur laquelle se développe la mélodie. C'est la métaphore de la vaine dispute entre méthode inductive ou méthode déductive. Le chercheur de terrain ne se contente pas de discuter de tout et de rien avec des individus rencontrés par hasard, en attendant que le terrain lui révèle son sujet. Trop souvent les discours sur la valeur de l'induction embrassent ce mythe d'une immédiateté quasi magique dans les interactions de terrain. Non, le chercheur approfondit son terrain à mesure qu'il maîtrise la grammaire de son champ de recherche. Il y a d'abord les lectures théoriques, par exemple pour moi sur la notion de citoyenneté, sur les modèles de " démocratie participative » etc. Puis, comme le musicien qui se nourrit des enregistrements et des concerts de ses pairs, le chercheur construit une connaissance des travaux similaires - ce qui, soit dit en passant, est une raison cruciale pour encourager les études de cas avant toute prétention à une montée en généralité universaliste. De même le reproche fait aux démarches déductives de s'enfermer dans un systématisme étroit ( une loi + un exemple d'application = un argument scientifique ») oublie que le langage scientifique nécessite un apprentissage aussi méticuleux que celui du musicien. Tout jazzman sait adapter son vocabulaire aux multiples circonstances : le phrasé, la rythmique ne sont pas les mêmes entre un standard de New Orleans ou un morceau de be bop... J'utilise bel et bien des mots différents selon que je tente de discuter avec un élu de la représentativité de son comité de quartier, que je m'adresse à une grand-mère pas très à l'aise en anglais, ou que je réponds à un avocat spécialiste des questions de logement qui s'interroge sur les enjeux de mon travail. Enfin, pour participer à une jam session, une improvisation collective, il faut aussi simplement négocier sa place physiquement : écouter les autres musiciens, savoir se taire, mais aussi savoir prendre la parole pour un solo sans hésiter afin de ne pas briser l'énergie du collectif... L'observation participante répond à tous ces défis. Lorsque je suis dans le salon de Faeeka pour célébrer le baccalauréat de sa fille, je ne peux pas seulement m'asseoir dans un coin pour prendre note du défilé des voisins. Mon tour vient aussi où je dois poser au bras de la jeune diplômée et sourire aux cinq téléphones portables brandis pour immortaliser ce moment dans le flash des caméras intégrées. Progressivement, les rôles s'inversent et se confondent. Je ne rends plus visite à Bonita, j'embarque toute la famille pour une balade en bord de mer, à l'occasion de laquelle Eugene me raconte ses années en prison. Après avoir appris à lire la musique, à interpréter une partition, le temps est venu d'exercer la mémorisation. Je noterai plus tard les passages cruciaux de ces récits libres et reposerai quelques questions factuelles lors de notre prochaine discussion s'il le faut. Le principe musical élémentaire 'de question-réponse', le fameux 'quatre-quatre', lorsque le batteur et le bassiste enchaînent des solos toutes les quatre mesures, devient le mode de fonctionnement dominant. Zaza me demande de venir participer à la réunion de dimanche pour rendre compte à tous les voisins de ce que l'avocat a expliqué mercredi soir. «Je n'ai pas très bien compris ce qu'il a dit à propos de notre fichage comme 'interdits bancaires', tu pourrais leur expliquer toi ?»... 
Allers-retours entre théorie et performance pratique, variations improvisées autour d'un thème pré-écrit, l'image de la jam session met l'accent sur la pluralité des voix qui composent l'œuvre scientifique. Idéalement, le terrain serait cet espace-temps à mi-chemin entre la répétition où l'on négocie l'équilibre des voix et le concert où l'on donne à voir un instant arrêté.

A partir de ce cadre commun à tous les arts fondés sur l'improvisation (danse, théâtre, tous ces arts que l'on qualifie de 'vivants'...), certains défendent des choix esthétiques divergents en insistant sur la valeur politique de l'improvisation. Il en est ainsi des fondateurs du free jazz, les premiers morceaux enregistrés par Ornette Coleman ou Cecil Taylor, les rencontres entre Archie Shepp, John Coltrane, Eric Dolphy ${ }^{4}$, réclament une émancipation du cadre trop étroit du jazz classique. L'ouvrage de P. Carles et J.-L. Comelli retrace les parentés entre le discours radical du mouvement 'noir' et le développement du free jazz aux Etats-Unis. En choisissant de développer ses improvisations entre les sentiers balisés de la théorie musicale, l'avant-garde musicale noire étatsuniennes a donné corps à des sons nouveaux, inventant un langage provocateur qui a fait voler en éclat le consensus souvent teinté de paternalisme qui pesait sur la musique noire réduite à un jazz de commande. Le free jazz s'est aliéné les critiques musicales mais a défendu une image nouvelle de la condition noire aux Etats-Unis. En assumant des arts-de-faire entre les normes définies par les manuels, en tentant une improvisation provocatrice, en trébuchant sur un langage métisse, il est peut-être possible de donner un autre souffle à une production trop souvent saturée par les conventions. C'est peut-être ce que propose l'équipe du colloque 'Terrain' en ouvrant la boîte noire de nos pratiques auto-proclamées scientifiques.

\section{Eloge de la sensibilité pour un discours scientifique 'émancipé'}

Trop souvent la réflexivité épistémologique et méthodologique est abordée comme un 'horstexte', réservé à la préface ou aux annexes du 'vrai' texte scientifique. J'espère avoir ici montré qu'une géographie sociale qui s'interroge sur son rapport au terrain est non seulement capable, au même titre que des approches classiques qui ne se confronteraient pas avec la 'boîte noire' du terrain, de produire du savoir mais également de nourrir une certaine ontologie de la recherche en science humaine.

Il est possible, voire nécessaire, d'intégrer le chercheur au tableau des situations sociales observées. Photographes et caméramans apprennent à maîtriser cette mise en abîme lorsque leur reflet apparaît dans le champ. De la même manière, le discours scientifique devrait être capable de se montrer comme un processus. Dans cette construction intellectuelle, le travail de terrain n'est pas une simple étape limitée dans le temps, il est un ingrédient constamment remanié. Le matériau brut est travaillé a posteriori dans le texte scientifique -comme par exemple avec l'interview de Rose, et ce faisant, il nourrit les interviews futures. Quand bien même, le chercheur 'change de terrain', c'est le même processus qui continue à travers les interactions dans d'autres villes, d'autres contextes, d'autres projets. Faire du terrain, c'est avant tout une façon d'être-au-monde, marqué par la sensibilité et l'empathie, avant que d'être adoubé par la reconnaissance institutionnelle de la Science.

"With an emancipatory interest, the philosopher apprehends given social circumstances not merely in contemplation but with passion : the given is experienced in relation to desire. Desire, the desire to be happy, creates the distance, the negation, that opens the space for criticism of what

4 La discographie proposée à la fin de l'article fait référence aux albums 'phares' les plus cités dans les études sur le free jazz. Il faut toutefois rappeler que l'idée même d'un 'courant musical' uniforme appelé free jazz est au cœur de nombreux débats musicologiques malheureusement pas développés ici. 
is. 》 (Young, $1990: 5-6$ ).

Iris Marion Young, philosophe, spécialiste de la notion de Justice, revient sur les racines idéologiques de l'engagement intellectuel. 'Le désir d'être heureux'. Se présenter sur 'le terrain' comme un simple être humain en quête de bonheur, voilà une posture bien romantique mais il est peut-être temps pour les sciences de prendre en compte leur valeur poétique à l'image de la sémiotique nouvelle explore par le free jazz. La quête improvisée affirme la valeur esthétique de la liberté 'grammaticale'. Au fondement de la démarche 'libre', repose la valeur absolue donnée à la rencontre, à l'instant. Une improvisation n'est pas limitée par une nécessaire cadence finale, elle crée elle-même son espace-temps, ses propres codes... C'est un modèle utopique pour le chercheur mais il faut bien de l'utopie pour continuer à défendre le désinteressement des sciences sociales et la possibilité de 'faire du terrain' comme façon de mettre en actes nos questionnements.

\section{Bibliographie}

CARLES P., COMOLLI J.-L., 2000, Free Jazz, Black Power, Paris, Poche

DE CERTEAU M., 1990, L'invention du quotidien, T. 1 : Arts de faire, Paris, Le Seuil

COLLIGNON B., 1996, Les Inuit, ce qu'ils savent du territoire, L’Harmattan

DELEUZE G., GUATTARI F., 1980, Capitalisme et schizophrenie. T. 2 Mille Plateaux, Paris, Ed. De Minuit

GERVAIS-LAMBONY P., 2003, Territoires citadins, 4 villes africaines, Paris, Belin

HOLSTON J., 2008, Insurgent Citizenship. Disjunctions of Democracy and Modernity in Brazil, Princeton/Oxford, Princeton University Press

LEFEBVRE H., 2000 (4eme ed.), La production de l'espace, Paris, Economica

YOUNG I. M., 1990, Justice and the politics of difference, Princeton Univ. Press

\section{Discographie}

Quelques références reconnues comme les 'classiques' du free jazz :

Ornette Coleman, 1960, Free Jazz, a collective improvisation, label Atlantic

John Coltrane, 1965, Ascension, label Impulse!

Eric Doplhy, 1964, Out to Lunch, label Blue Note

Cecil Taylor, 1966, Unit Structure, label Blue Note 